\title{
Using General Collective Intelligence to Re-think Decision-Systems and Other Information Technologies \& Information Systems to Better Inform Pandemic Responses
}

\author{
Andy E. Williams, Nobeah Foundation, Nairobi, Kenya
}

\begin{abstract}
:
The emerging science of Human-Centric Functional Modeling uses functional state spaces to provide a completely self-contained representation of human perceptions in the functional domain of each consciously observable human system (body, emotions, mind, consciousness). In the case of the cognitive system (mind) this constitutes a complete semantic model of information. This semantic model is required to implement a General Collective Intelligence software platform hypothesized to be capable of acting as a collective cognition that significantly increases capacity for group problemsolving through collective reasoning. This paper explores how the lack of this General Collective Intelligence based decision-making platform, and hence the lack of this semantic modeling, might be a systemic error in all group decisions, including policy decisions, with the result of preventing governments from achieving optimal outcomes with regards to the COVID-19 pandemic. This paper also explores why this newly emerging science of General Collective Intelligence or GCI as a model of collective decision-making is predicted to radically increase capacity to solve collective problems, and why this is predicted to radically impact COVID-19 response policy.
\end{abstract}

Keywords:

COVID, General Collective Intelligence, Human-Centric Functional Modeling, system dynamics, functional state space

\section{Introduction}

A General Collective Intelligence or GCI [1] is a hypothetical platform that self-assembles individuals in a self-sustaining way into potentially massive networks of cooperation able to execute collective reasoning with general problem-solving ability, and therefore problem-solving ability that might be targeted at solving any problem in general. In this general problem-solving ability those networks of cooperation might be leveraged to achieve a predicted exponential increase in outcomes of that collective reasoning [2].

By analogy with the human organism, in order to intelligently respond to any bodily ailment, it is necessary to have both a sensory-motor system to detect the state of the body, and a cognitive system to reason out a course of action. Through this analogy we can see that one key challenge with COVID related public health policies is the lack of an analog to the sensory-motor system that enables us to determine the "state" of collective health. In Human-Centric Functional Modeling (HCFM) a system is represented as having a set of processes through which it might transition between different functional states. The behavior of the system then moves through a graph containing a network of nodes in which each node represents a functional state, and each edge between states represents a process by which one functional state might transition to another. This graph is referred to as a "functional state space".

Our sensory-motor system also moves through different functional states as functions or processes within the sensory-motor system occur. This transition between sensory-motor states occurs through our conscious execution of motor processes or when the execution of our sensory reception processes is triggered and we consciously detect sensations. Each system within the human organism is represented as interacting dynamically with its environment and with the other human systems. Each process in each human system is represented in HCFM as potentially having a different "fitness" with which it might achieve its targeted outcome. This fitness might be used by the sensory-motor system (body) to 
compare one potential process that might be executed, with another. The fitness of the sensory-motor system to achieve all of its functions is represented as the level of physical or bodily well-being, which is in turn represented as a position in a "fitness space". Since the sensory-motor space is a composition of tactile, visual, auditory, gustatory, and olfactory spaces, this bodily well-being is a composition of well-being in the tactile, visual, auditory, gustatory, and olfactory systems as well. In addition to fitness in the body, the human organism is represented in HCFM as also having fitness in the emotions, mind, and consciousness. These systems (body, emotions, mind, and consciousness) form a composite "human state space" that the individual moves through. Any current metrics used to determine the state of our collective health are incomplete in that unlike our human state space, they cannot incorporate all possible factors impacting our well-being.

In addition, existing metrics are not only incomplete but also potentially biased, as the choice of which metrics to include and which to leave out can easily become aligned with the perspective or ideology of the decision-maker involved [3]. Another key challenge is that without special measures that do not appear to be taken in any existing groups today, there are a significant number of COVID related problems that group decision-making cannot solve.

As mentioned, the emerging science of Human-Centric Functional Modeling on the other hand can potentially be used to define a "human state space" that incorporates not just the state of the body, but also the state of the emotions, the mind, and the self-conscious awareness (consciousness). This solves the problem of the lack of a more holistic means to track the complete state of public health. HumanCentric Functional Modeling can also be used to define a General Collective Intelligence platform able to act as a collective cognition hypothesized to have the ability to reliably address the problems in group problem-solving that prevent COVID related public health policies from being optimized.

\section{Lack of a coherent collective intelligence capable of switching between type 1 and type 2 reasoning}

In the absence of GCI, any group suffers from systematic errors in decision-making that limit its collective intelligence. As an example, what is commonly lost in discussions about what COVID policy is best is that there are two main cognitive biases individuals might have (type 1 or intuitive reasoning and type 2 or rational methodical reasoning), neither of which permits an understanding of the other. In other words, type 1 arguments can't be used to convince a person who is using type 2 reasoning, and vice versa. Both reasoning types are useful, but for solving different types of problems. Societies always have some individuals biased towards type 1 reasoning and some individuals biased towards type 2 reasoning. Both biases are useful, but without a platform such as GCI to help select the optimal bias in each context we cannot as groups switch between these biases in an optimal way, the same way we can switch between these biases within our individual cognition. Furthermore, we lack a comprehensive model able to take into account all possible factors affecting the well-being of the population so that more intelligent decisions can actually be made.

What is the evidence that there is no coherent collective intelligence capable of switching between type 1 and type 2 reasoning and that type 1 and type 2 reasoning come to different conclusions that can't be resolved? Assuming (as predicted) that type 1 and type 2 reasoning are each associated with predispositions towards different political ideologies, it would be expected that polarization between political ideologies should be increasing, and that the impact of exposure to opposite opinions should increase this polarization. That is exactly what we appear to see [4], [5], [6], [7], [8].

\section{Lack of Sufficient Collective Intelligence to Reliably Solve Wicked Problems of Public Health Policy}


Understanding that there is insufficient collective intelligence with regards to COVID policy begins with identifying and confirming the number of problems related to COVID policy that groups currently can't reliably solve. A list of some of these problems is provided in table 1 .

\section{Problem Name Description}

Alignment of Choices The problem of misaligned incentives. In many cases we not only tend to solve with Individual Interests the wrong problem, but also tend to be constrained to do so. Solving this problem of misaligned interests requires decentralized decision-making that can't become aligned with any centralized interests.

Better Choices are Not The problem of the inability to compare options and reliably select best one. An Useful example is "The Problem of Finding the Better Mousetrap". The solution to radically increasing collective impact is often not "building a better mousetrap". Instead the solution is building a system of decision-making capable of reliably selecting and supporting the best mousetrap. In addition no decision system can improve things if it isn't used. Solving this problem also requires a system of organization that out-competes other systems so that it is reliably used.

Shortcomings of Systems Science Methodologies are Invisible

Lack of Coherence in Group Reasoning

The Problem of Direction
There are a number of patterns of problems in applying systems thinking that anecdotal observation suggests are very prevalent. One being confined to type 1 (fast or intuitive) reasoning which determines truth or falsehood using intuition, which relies on patterns of solutions observed in the past, one of which is group consensus. However, type 1 reasoning is incapable of solving problems requiring that new reasoning algorithms (e.g. new logic or new methods of calculation) be used since no consensus can exist for any method that is new. Another is being confined to type 2 reasoning where reasoning algorithms are incorrect or cannot successfully be executed to completed. Where the preferred reasoning method is assumed to be correct these errors are invisible.

Individuals have both type 1 (fast or intuitive) reasoning and type 2 (rational methodical or slow) reasoning. Type 1 reasoning is necessary for solving uncomputable problems, and type 2 reasoning is necessary in solving computable ones. Type 1 and type 2 reasoning come to different conclusions, but individuals can switch between type 1 and type 2 reasoning depending upon which is optimal. Therefore individuals can reliably converge on a conclusion. However, some individuals are prone to type 1 reasoning in certain circumstances in which other individuals are prone to type 2 reasoning. This leads to polarization in groups. Without a "collective cognition" that is able to switch the group from type 1 reasoning to type 2 reasoning or vice versa, groups cannot reliably converge on optimal collective reasoning. In this sense groups without collective cognition lack coherence in group reasoning. The problem of the inability to discern which direction provides the most productive path forward in the case of wicked problems. Where an interaction between three components of a system is results in some function that solves a problem, that problem might be reliably definable, and that solution might be reliably discoverable. On the other hand, where the interaction is between one thousand components of the system, that problem might not be reliably definable and the function providing the solution might not be reliably discoverable. Such chains of cooperation between activities required to define or to solve the problem represent a path in some "functional state space" that is too long and therefore too complex. These chains of cooperation might radically increase whatever collective outcome is being targeted, but in order for there to 
The Problem of of Cooperation Complexity in Chains

be a clear direction forward towards identifying opportunities for such cooperation, there must be a more general way of assessing impact so that the impact of different directions forward (i.e. the predicted impact of one potential chain of cooperation vs another) can be compared.

The problem that complex cooperation is required to radically increase ability to achieve collective outcomes, but cooperation becomes more unlikely the more complex it becomes. If a chain of 5 projects is very difficult to launch, a chain of 10,000 projects might be impossible. Finding chains of cooperation that exponentially increase outcomes is only part of the problem. The problem is also decoupling the chains so participants only need to understand their link in the chain in order to decide whether to participate. This problem of inability to enter into chains of cooperation that are too complex has implications regarding the length of reasoning, and therefore the complexity, that models of GCI (General Collective Intelligence) can navigate, wherever those models are based on decentralized components that cooperate to achieve an outcome. This also determines which problems cannot reliably be solved (i.e. it determines which problems are "wicked problems").

Table 1: Some of the holes in decision-making (problems that cannot reliably be solved and solutions that cannot reliably be discovered) without General Collective Intelligence.

If solutions are defined in conceptual space as reasoning which navigates a path from one concept to another, these decision-making problems represent gaps in the collective conceptual space in the sense that collective reasoning can't reliably navigate to these regions.

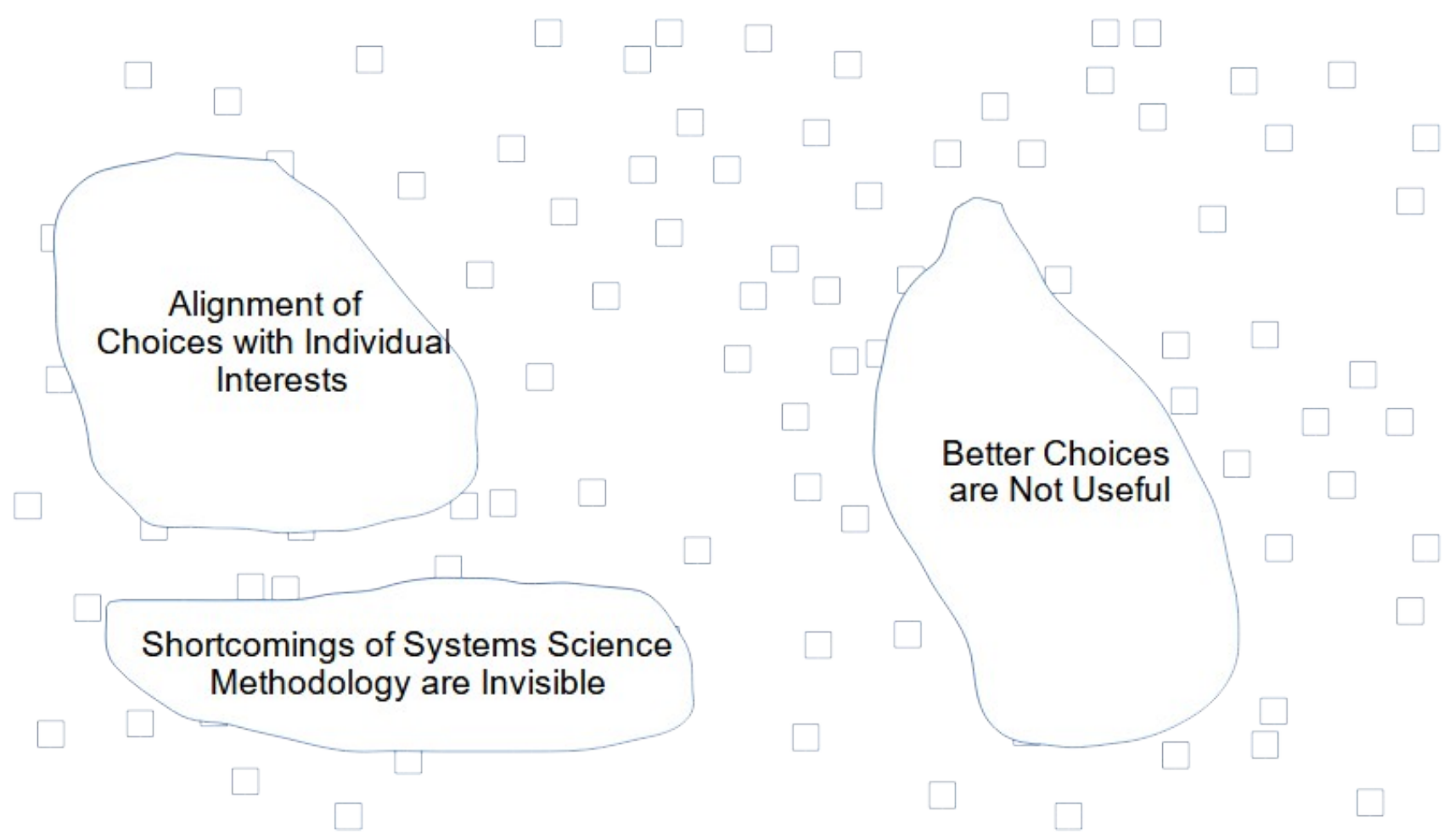

Figure 1: Gaps in the collective conceptual space which collective reasoning can't reliably navigate, representing problems that groups can't reliably solve and solutions that groups can't reliably discover. 
What is the evidence that there is currently insufficient collective intelligence to reliably solve wicked problems of public health policy? One is that the inflexibility implied by polarization reflects a lack of sufficient intellectual capacity in managing outcomes [9]. Another is that if ability can be insufficient with respect to a targeted outcome, then lack of that ability can define a disability. Intellectual disabilities in individuals are observed to exist [10]. The definition of intellectual disability as representing "significant limitations in both intellectual functioning and adaptive behavior" would appear to clearly apply to any current group lacking GCI, if GCI as predicted, is able to reliably achieve an exponential increase in the general problem-solving ability of groups, as measured by the collective intelligence factor (c).

\section{Lack of Sufficient Collective Consciousness to be Aware of These Problems}

It might be intuitively clear to most that awareness of the problems involved in coming up with better COVID policies is essential in solving those problems, but what is less obvious is how to come up with a common definition for what exactly awareness is, and how to quantify it. The brain, which is to date the most sophisticated device known by man to exist anywhere in this universe, can at this point only be understood by first person observation of its functions, since there are not yet any external tools capable of detecting the existence of specific concepts or the execution of reasoning within the brain. However, there is not yet any science capable of treating first person observation as anything but subjective and non-scientific. Therefore the vast majority of observations that might be made are excluded from consideration. This inability to properly consider first person observation is true not just for the human mind, but for the entire biology of this human organism, including consciousness. Researchers such as Varela attempted to bridge this gap through defining a "first-person science" he called neurophenomenology [11], but the newly emerging science of Human-Centric Functional Modeling or HCFM goes a few critical steps further. In this science, all of human perception is represented in terms of fields within "functional state spaces". These functional state spaces enable all the properties of first person observation to be quantified. For example, cognition can be represented in terms of the mind using reasoning to navigate a space of concepts or a "conceptual space", that serves as the functional state space of the mind. In this functional state space, truth is a property that has a well-defined representation, so the truth of any first person observations can be objectively assessed, thereby making first person observations accessible to science for the first time.

Human-Centric Functional Modeling represents the magnitude of an individual's intelligence in terms of their ability to search a given volume of "conceptual space" per unit time in order to find the solution to a conceptual problem. HCFM also represents human consciousness as directing the focus of attention or awareness, and represents the magnitude (or level) of an individual's consciousness in terms of their ability to search a given volume of "awareness space" per unit time in order to find an awareness that allows problems to be perceived in the first place [12]. HCFM suggests both relative and absolute truth are represented by well defined patterns in the conceptual space, and therefore suggests that truth has an objective definition that can be perceived through first person observation of the individual's own cognitive system (mind). Since the functional state spaces of the cognition and of the consciousness are hypothesized to share a common representation, this suggests the same pattern might be used to identify truth in the consciousness system (in the conscious self-awareness). The ability to distinguish truth from falsehood in any perceived awareness of whether all possible parts, some parts, or no parts of a problem or solution are within or can be brought within our awareness, might be far more important than having the intelligence to solve the problem. Stated in terms of common wisdom, there are those who know, those who can be shown, and those who cannot be told. It is the capacity for awareness (level of consciousness) rather than capacity for problem-solving (intelligence) that might separate the three. 
What is the evidence that there is insufficient collective consciousness to be aware of these problems? Again, if ability can be insufficient with respect to a targeted outcome, then lack of that ability can define a disability. Deficits of awareness in individuals, particularly those who have suffered stroke or other injuries, are observed to exist [13]. Whether or not any current group lacking GCI would have a significant "deficit of awareness" as compared to a group organized by a GCI remains to be confirmed.

\section{Validating the Hypothesis of Optimal COVID Policy Being a Pattern in Functional State Space}

If optimal COVID decision-making is a pattern in some functional state space how is that space defined and what does that pattern look like? Is optimal COVID decision-making a pattern in the collective conceptual space that serves as the functional state space of the collective cognition? Is optimal COVID decision-making a pattern in some functional state space (i.e. the "human state space") that defines the state of each individual in the group? Though it is still very powerful in its current form, the idea of conceptual space is incomplete. There are a number of problems that must be solved before any implementation of a conceptual space can be used to semantically store concepts and reasoning that might be searched to find solutions to COVID decision-making problems. One is that distances are not precisely quantified. Another is that there is not yet a representation of concepts and reasoning relationships that is fully self-contained (independent of any lookup table). The same applies for the human state space. In the mean time we must use the subset of conceptual space and the subset of the human state space that can be represented with ontologies, and we must accept the limitations of working with ontologies as opposed to working with a complete semantic representation. However, because any functional state space might be represented by a graph containing a network of nodes representing functional states connected by edges representing the processes through which the system transitions between states, then solving these problems for any functional state space solves these problems for every functional state space, including the conceptual space.

\section{Navigating the Gaps in Conceptual Space Related to Sub-Optimal COVID Policy}

In the current designs approximating GCI platforms, collective reasoning problems concerning how to achieve collective outcomes targeted by the collective reasoning of a group (problems requiring collective reasoning processes to be directed at achieving specific collective outcomes) are approximated as one of three types: closed problems solvable with chains of collective reasoning involving existing concepts, closed problems solvable with chains of collective reasoning involving new concepts, and open problems solvable with new generalizations of concepts or more detailed specialization of concepts that are incorporated into new concepts used in chains of collective reasoning [14]. Classifying these reasoning problems into these patterns enables all solutions to be readily compared in terms of their projected impact on the targeted outcome using the same general method of comparison for each pattern and outcome.

An example of the first pattern is the proposed "Social Impact Marketplace" platform in which the goal is to orchestrate cooperation to define value chains of businesses that cooperate to meet local demand while vastly increasing collective social impact through one business agreeing to buy a key product or service from one business in the value chain in return for another business in the value chain agreeing to buy from them. In this example the product or service exchanged between one business and another represents the "concept", and the set of interacting businesses represent the chain of cooperation that is conceptualized by a chain of collective reasoning. Classifying these reasoning problems into these patterns not only enables all solutions to be readily compared in terms of their projected impact on the targeted outcome using the same general method of comparison for each pattern and outcome, but also enables all individuals in a group to collaborate in suggesting such solutions, and enables all individuals to collaborate in evaluating which solution is predicted to be the best. Because solutions can be defined, objectively compared, and selected by exponentially more people, rather than being 
defined, subjectively compared, and selected by only a few people, then the ability to search an exponentially greater number of such solutions in order to select the best one can be demonstrated. It is hypothesized that this ability to search an exponentially greater number of solutions might equate to the ability to search an exponentially greater region in the collective conceptual space.

Each of the three patterns of solution is represented as a set of concepts that are described by an ontology. This ontology forms some subset of the conceptual space defined by Human-Centric Functional Modeling. The many instances of proposed solutions that are defined within that ontology form an even larger subset of the conceptual space, so that by defining more and more problems with these patterns, and by discovering more solutions with these patterns, a larger and larger subset of the conceptual space might be represented.

Human-Centric Functional Modeling represents collective reasoning as navigating the collective "conceptual space" in a way that leads to general problem-solving ability for the group. Each reasoning process has a projected fitness in achieving some targeted function, as well as a target fitness in achieving that function, and an actual fitness in achieving that function. This projected, targeted, and actual fitness defines a general "fitness space". General problem-solving ability is represented as the potential ability to navigate the entire collective conceptual space in a way that is dynamically stable in this "fitness space" that describes the fitness of the cognitive system to execute all of its reasoning functions ("cognitive fitness"). This dynamic stability is achieved through ensuring that in selecting and executing reasoning processes the cognitive system follows a set of equations that are stable in the three dimensions of fitness space. One set of equations which exhibit dynamical stability in three dimensions and which might be used to approximate such an intelligence algorithm are the Lorenz equations for convection [15].

In the "Social Impact Marketplace", which is proposed to be the first implementation of GCI, the conceptual space is approximated by three ontologies, with each ontology representing one of the three patterns of solution by which sustainability or other impact might be exponentially increased. For each pattern of solution the platform automates the collaboration required to select the value chain that maximizes targeted outcomes. In doing so the algorithm creates the ability to overcome the limitations in group problem-solving ability representing the gaps in the collective conceptual space that can't reliably be navigated today. To do so the GCI navigates the conceptual space with an algorithm that is intended to approximate general problem-solving ability. Take the case in which $\mathrm{N}$ potential participants in cooperation must select between $\mathrm{M}$ processes of cooperation, where $\mathrm{M}$ is greater than $\mathrm{N}$. Or more specifically, the case in which $\mathrm{N}$ potential participants must select between $\mathrm{M}$ value chains of businesses. Centralizing the decision of which chain of cooperation (value chain) will be used so that it is made by any single individual might align the decision with that individual's interests, rather than with maximizing collective fitness. Such centralization would then represent individuals reasoning separately within a group, rather than representing collective reasoning. The collective reasoning process in this platform requires each individual to suggest a solution to achieving a targeted outcome, where that solution fits one of these three patterns. The platform then enables each individual to bid to participate in some chain of cooperation proposed by any individual on the platform. The individual with the first role in the chain of cooperation selects the top $\mathrm{N}-1$ chains of cooperation from their perspective, the second individual selects the top N-2 chains of cooperation from their perspective, and so forth until the $\mathrm{Nth}$ individual selects the final chain of cooperation that all $\mathrm{N}$ actors participate to execute. This algorithm is predicted to result in outcomes that are maximally aligned with the interests of all. This algorithm is very different from direct democracy or other democratic voting processes because it identifies processes predicted to achieve optimal outcomes, rather than identifying an individual or other entity who is then free to select outcomes that are aligned with their own interests. 
General Collective Intelligence is bio-mimicry of the way nature designs organisms, and nature designs cooperation within organisms in a way that is democratic from a distributed and decentralized point of view, but not democratic from a centralized perspective. In other words, cells in the brain and cells in the bottom of the feet, or cells in the left and vs cells in the right hand, don't both vote together in the same processes, and no cell is elected ruler over all others.

\section{Discussion and Directions Forward}

From the perspective of this approach it is hypothesized that solving "wicked problems" in health care are problems of higher order complexity that cannot reliably be solved without higher general problemsolving ability. A GCI platform is hypothesized to create the potential to exponentially increase the general problem-solving ability (intelligence) of the group over that of any individual in the group [2], so that such problems might become reliably solvable.

However, increased collective intelligence applies to ability to execute a single decision. The vastly increased number of decisions expected to accompany GCI must also be tracked. System dynamics has long been used to model the interplay of a number of factors in the behavior of complex systems. The natural next step is to explore how General Collective Intelligence might eventually be combined with system dynamics to create models representing the impact of health care policies on collective wellbeing using a collective "human state space". The directions forward are then:

- To define a minimal ontology to represent the state of the human system related to COVID, where that ontology might be elaborated over time into a "human state space" capable of representing the collective health state of populations.

- To define a simple decision-making platform with an approximation of general problem-solving ability in the domain of a few key COVID related healthcare policy decisions, where that platform might be elaborated into a General Collective Intelligence over time.

- To create a simple implementation of a simulation that leverages systems dynamics to simulate the interaction of factors relevant to COVID in the population, where that simulation might evolve over time into a "cognitive simulation platform" able to leverage General Collective Intelligence and the collective human state space to model the impact of any health care, social welfare, or other public policy.

\section{Conclusions}

This paper has explored a number of problems in the formation of public health policy including:

- Lack of a coherent collective intelligence capable of switching between type 1 and type 2 reasoning.

- Lack of sufficient collective intelligence to reliably solve wicked problems of public health policy.

- Lack of sufficient collective consciousness to be aware of these problems.

Many other researchers have explored the use of models to represent the state of public health. However there is no model comprehensive enough to provide the collective ability to model the impact of all factors relevant to COVID, whether depression from isolation due to lock downs, whether the differing mortality rates for different age groups, or all other factors. This is something that HumanCentric Functional Modeling hopes to address through defining a collective "human state space". While this is a massive task, the powerful implications of this model in every branch of health and wellness mean that there is also a massive number of potential beneficiaries of the effort who might be incentivized to participate in defining such a model. Other researchers have also explored the use of collective intelligence to improve health care related decision-making. But this paper, along with other existing published work, have also identified some current errors in group decision-making errors that no individual and no current collective intelligence that is not GCI can reliably solve, and that result in 
the inability of groups to reliably converge on a single optimal line of reasoning. Other published work has explored how General Collective Intelligence might resolve this current inability to optimize decision-making [16].

To whatever degree that the response to the pandemic was sub-optimal, that error might have cost billions in terms of impact on human well-being. GCI might be the single most important issue with regards to COVID, since according to this model it's not possible for groups to reliably benefit from better information in a number of very well-defined circumstances in which even the best research published in peer-reviewed articles simply doesn't reliably result in improved public health policies. This model predicts that systemic error in the decision-making of groups that aren't organized by a General Collective Intelligence can't be solved regardless of the amount of discussion or regardless of the amount of information on the topic that is shared.

\section{References}

[1] Williams, A. E., Defining a Continuum from Individual, to Swarm, to Collective Intelligence, to General Collective Intelligence, International Journal of Collaborative Intelligence, in press (2022)

[2] Williams, A.E. (2021) Are wicked problems a lack of general collective intelligence?. AI \& Soc (2021). https://doi.org/10.1007/s00146-021-01297-8

[3] Gregg Henriques, Andy E. Williams, General Collective Intelligence and Systemic Error in Groups, retrieved from https://www.psychologytoday.com/us/blog/theory-knowledge/202106/generalcollective-intelligence-and-systemic-error-in-groups, on July 19, 2021.

[4] Bail, Christopher A., et al. "Exposure to opposing views on social media can increase political polarization." Proceedings of the National Academy of Sciences 115.37 (2018): 9216-9221.

[5] Garrett, R. Kelly, et al. "Implications of pro-and counterattitudinal information exposure for affective polarization." Human communication research 40.3 (2014): 309-332.

[6] Levy, Ro'ee. "Social media, news consumption, and polarization: Evidence from a field experiment." American economic review 111.3 (2021): 831-70.

[7] McCright, Aaron M., Chenyang Xiao, and Riley E. Dunlap. "Political polarization on support for government spending on environmental protection in the USA, 1974-2012." Social science research 48 (2014): 251-260.

[8] Fiorina, Morris P., and Samuel J. Abrams. "Political polarization in the American public." Annu. Rev. Polit. Sci. 11 (2008): 563-588.

[9] Babío, Nuria Calvo, and Rafael García Rodríguez. "Talent management in professional services firms: A HR issue?." International Journal of Organizational Analysis (2010).

[10] Schalock, Robert L., et al. Intellectual disability: Definition, classification, and systems of supports. American Association on Intellectual and Developmental Disabilities. 444 North Capitol Street NW Suite 846, Washington, DC 20001, 2010.

[11] Varela, Francisco J. "Neurophenomenology: A methodological remedy for the hard problem." Journal of consciousness studies 3.4 (1996): 330-349. 
[12] Williams, A. E. (n.d.). Human-Centric Functional Modeling and Defining Metrics for the Function and Level of Consciousness. Retrieved from osf.io/preprints/africarxiv/28vty

[13] Flashman, Laura A., and Thomas W. McAllister. "Lack of awareness and its impact in traumatic brain injury." NeuroRehabilitation 17.4 (2002): 285-296.

[14] Williams, A. E. (2022, February 27). Using Models to Capture Patterns for Radically Increasing Social Impact. https://doi.org/10.31730/osf.io/rqs72

[15] Williams, A. E. Approximating an Artificial General Intelligence or a General Collective Intelligence, International Journal of Collaborative Intelligence, in press (2022)

[16] Williams, A. E., Is General Collective Intelligence a Reliable Path Towards Achieving Green Growth?, International Journal of Green Economics, in press (2022) 